\title{
O USO DA WEBCAM NA EDUCAÇÃO
}

Maurício José Viana Amorim, PGIE/UFRGS, IFF Campos-RJ, amorim@iff.edu.br

Magda Bercht, PGIE/UFRGS, bercht@inf.ufrgs.br

Resumo: Este artigo apresenta algumas formas de utilização das câmeras de vídeos acopladas a computadores, as webcams, na educação sob a ótica do apoio ao professor, aos processos pedagógicos e de apoio ao aluno. Discute o uso de tal tecnologia para a verificação da motivação entre outros aspectos afetivos. A base que fundamenta esta discussão está no fato de que os estados afetivos do aluno podem contribuir com a aprendizagem. $O$ modelo de reconhecimentos de emoções através do mapeamento de expressões faciais proposto por Ekman é apresentado e sua aplicação nas áreas da Computação Afetiva e da Educação é observada sob o ponto de vista das tecnologias envolvidas e principalmente das perspectivas que esta forma de interação pode suscitar.

Palavras-chave: Computação Afetiva, Reconhecimento de Emoções em Expressões Faciais, Educação.

\section{The use of the webcam in Education}

\begin{abstract}
This paper presents some ways of using video cameras coupled with computers, the webcams, in education from the perspective of teacher support, pedagogical process and student support. It discusses the use of such technology for the verification of motivation and other affective aspects. The fundament about this discussion is based on the fact that affective states can contribute to the learning. The model of recognition of emotions by mapping facial expressions proposed by Ekman is presented and the application in Affective Computing and Education are observed from the point of view of the technologies involved and especially the outlook that such interaction can generate.
\end{abstract}

Keywords: Affective Computing, Recognition Emotions in Facial Expressions, Education. 


\section{Introdução}

Quando pensamos em uma câmera de imagens para a web, webcam, como será utilizado neste artigo, logo nos vem à cena uma pessoa conversando e visualizando o seu interlocutor através de programas de mensagens instantâneas como o MSN e Skype ${ }^{1}$. As redes pessoais de mensagens instantâneas são de longe a aplicação mais popular que utiliza a webcam. Essa popularização tornou-se possível através do aumento da capacidade dos links com a internet e o barateamento dos valores cobrados pelas operadoras pelos links. Com links mais velozes, novas mídias podem ser utilizadas na comunicação, entre elas a voz e o vídeo.

O uso dessas mídias na organização e nos processos organizacionais e gerenciais das instituições de ensino já é uma realidade. Em (MSNBC, 2009) é relatado uso dos programas de mensagem instantânea com webcam para entrevistas de admissão em diversos cursos de pós-graduação e graduação das universidades americanas. Uma das universidades, a de Wake Forest relata que o uso desta tecnologia evita que o candidato tenha que arcar com os altos custos de uma viagem para efetuar a entrevista.

Mas os programas de mensagem instantânea não são os únicos beneficiados com esta nova realidade. A educação e principalmente, em sua vertente -a distância (EAD), entra em uma nova era, onde o uso da webcam tende a ser um dos vértices tecnológicos. Vários projetos no mundo estão sendo criados, explorando a tecnologia das webcam na educação (Penteado \& Marana, 2009) (UEPG, 2009). As áreas de segurança, acessibilidade (portadores de necessidades especiais) e hipermídia adaptativa são exemplos de áreas que se beneficiam com pesquisas ligadas ao uso de webcam e educação (Bruce \& Young, 1986) (Brusilovsky, 1996) (Whitehill et al, 2009).

Neste artigo iremos desenvolver um estudo sobre o uso da webcam na perspectiva de apoio ao professor e aos processos pedagógicos, de verificação da motivação e outros aspectos afetivos. Ele baseia-se no fato de que os estados afetivos podem contribuir com a aprendizagem (Vigotsky, 1999, Goleman, 1995; Vail, 1994; Piaget, 1989) e na hipótese da webcam ser um dos meios eficientes, eficazes e não invasivos para a observação do sujeito enquanto aluno em desenvolvimento das tarefas, à semelhança das ações de um professor, em sua atenção ao aluno em uma aula presencial.

\section{Formas de uso do webcam na educação}

\subsection{Webcam e segurança na educação}

$\mathrm{Na}$ área de segurança aplicada à educação, as pesquisas tratam primordialmente de tentar identificar se o usuário que está realizando uma determinada tarefa é realmente quem ele diz. Um exemplo claro desta necessidade é na modalidade de EAD, em um processo de avaliação, uma webcam identificar se é realmente o próprio aluno quem está realizando a tarefa. Outro exemplo é o lançamento das notas e conceitos pelos professores que poderiam utilizar a webcam com critério extra de segurança.

Nesse contexto, (Penteado \& Marana, 2008) apresentam um sistema de reconhecimento facial de usuários de educação a distância por meio de webcam. A solução segundo eles "é para controlar as presenças dos alunos de e-learning podendo 
impedir, por exemplo, que outra pessoa fizesse uma avaliação no lugar do estudante matriculado" (Penteado \& Marana, 2009 pag.1)

O reconhecimento facial é uma área onde os projetos se concentram em conseguir métodos e sistemas cada vez mais eficazes, tanto em acurácia quanto em desempenho. Nessas áreas podemos citar as pesquisas de (Marin, 2009) e (Bruce \& Young, 1986). Os maiores desafios do reconhecimento facial são o fato da imagem do rosto nem sempre estar em posição frontal, à baixa resolução da imagem, a oclusão de partes da face e a dificuldade de localizar certas características relevantes.

\subsection{Webcam e os portadores de necessidades especiais}

Outra área onde a utilização da webcam pode ser útil é na ajuda aos portadores de necessidades especiais. Nesta área a webcam pode ser utilizada de diversas maneiras, apresentaremos abaixo algumas destas.

Em pessoas com dificuldades motoras, uma webcam pode ser utilizada para mapear o seu foco de visão e a partir de ações coordenadas, como por exemplo, piscar rapidamente o olho esquerdo e depois fechar o direito, simular as ações de um mouse e/ou de um teclado possibilitando estas pessoas interagirem com o computador e através dele com o mundo (Vieira \& Fontana, 2008).

Outra aplicação para o uso de webcam é o apoio a indivíduos com baixa ou nenhuma visão. Pesquisa muito interessante é a utilização de webcam como leitor braile. Pesquisadores da Universidade Estadual de Ponta Grossa (UEPG-PR) desenvolveram um protótipo que pode traduzir os sinais táteis da linguagem em alto relevo para algarismos da escrita convencional. De acordo com os pesquisadores, o grande mérito do projeto é associar facilidade de uso, simplicidade e baixo custo. "O número de estudantes com deficiência visual nas universidades brasileiras é maior do que você imagina", disse o vice-reitor da UEPG. No entanto, poucas são as instituições que estão preparadas para atendê-los - e menos ainda os professores que sabem ler braile (UEPG, 2009).

\subsection{Webcam e a computação dirigida por gestos}

Na chamada computação por gestos, uma câmera observa os movimentos do usuário, realizando ações quando certos padrões de movimento são captados. (Lyra et al, 2007) construíram jogos educativos para serem utilizado na reabilitação de pacientes de forma dinâmica e divertida. Os usuários realizam movimentos com o corpo na frente da câmera, estes movimentos são captados e estas ações/reações dos usuários se fazem sentir como forma de interação com o jogo.

\subsection{Webcam e a Hipermídia Adaptativa}

A chamada Hipermídia Adaptativa é outro ramo da informática na educação que pode ser bastante explorada pela tecnologia das webcams. Para explorar o tema iniciamos pela definição de Brusilovsky.

"Chamamos Sistemas Hipermídia Adaptativa (HA) todos os sistemas de hipertexto e hipermídia que absorvem algumas características do usuário em um modelo e aplica este modelo para adaptar-se e alterar diversos aspectos visíveis do sistema." (Brusilovsky, 1996 pag. 2).

Para adaptarem-se às expectativas, necessidades, preferências ou desejos dos usuários, a HA deve dispor de mecanismos para percebê-los. Uma das formas dessa percepção é o sensoriamento através da webcam. Através desta podemos perceber 
fatores como a velocidade de leitura, o grau de atenção, etc, e assim adequar a exibição dos conteúdos ou oferecer ajuda. (Whitehill et al, 2009) mostra um estudo da Universidade de San Diego no qual a webcam é utilizado como um "controle remoto". Eles realizaram um experimento no qual observando a face dos usuários, enquanto estes assistiam a uma palestra gravada, predizendo a velocidade de exibição no qual o usuário gostaria de assistir.

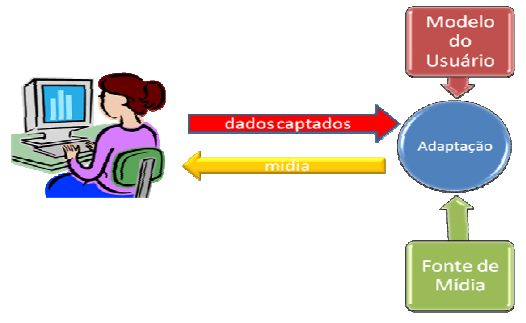

Figura 1- Sistemas de Hipermídia Adaptativa

Para empregar determinada técnica, os sistemas adaptativos buscam identificar padrões no modelo do usuário que justifiquem a execução de determinada função de adaptação (Nozawa et al, 2009; Jaques \& Vicari, 2008). Estes padrões são inferidos através do sensoriamento. O sensoriamento é a forma com que um sistema adaptativo reconhece fatos e crenças os quais serão utilizados para a tomada de decisões. Quanto melhor a forma de sensoriamento, melhor a ação realizada pelo sistema. O destaque dos aspectos afetivos nas atividades cognitivas não é recente. No início do século passado, Vigotsky já atentava para a importância da influência de fatores motivacionais na cognição (Vigotsky, 1999). Outros consagrados pesquisadores também destacaram estes aspectos (Goleman, 1995; Vail, 1994). Piaget (1989) enfatiza o incontestável papel perturbador ou acelerador da afetividade na aprendizagem. Estes estudos conquistaram respaldo no plano das Ciências Cognitivas a partir das pesquisas recentes como (Damásio, 1996; Le Doux, 1998).

\section{A Computação Afetiva}

A Computação Afetiva é um ramo da Inteligência Artificial que estuda as emoções. Picard (1997) define Computação Afetiva como "computação que está relacionada com, que surge de ou deliberadamente influencia emoções". Ele a subdivide em dois ramos maiores de pesquisa:

- Emoções na interação homem computador - onde visa reconhecer emoções humanas e/ou expressá-las;

- Síntese de Emoções - investiga a simulação de emoções em máquinas (síntese de emoções) a fim de descobrir mais sobre emoções humanas.

A pesquisa sobre emoção na área de Informática na Educação situa-se principalmente no primeiro ramo. Sistemas tutores inteligentes, agentes pedagógicos, hipermídia adaptativa tem sido algumas das de suas implementações.

Para que estes sistemas possam adaptar-se ao aluno ele deve ser capaz de reconhecer as suas emoções. Através dessa realimentação, o sistema pode perceber se o aluno está apto ou motivado a determinada atividade, realizar ações de modo a encorajá$1 \mathrm{o}^{2}$ a estudar.

\footnotetext{
2 Tarefas dos Agentes Pedagógicos Animados
}

V. $7 \mathrm{~N}^{\mathrm{o}}$ 3, dezembro, 2009 


\section{Sensoriamento de aspectos afetivos}

Quando pensamos no reconhecimento de estados afetivos, surge a necessidade de dispor de interfaces adequadas para captar os sinais emitidos pelo usuário para em seguida processá-los estes sinais e conseqüentemente atribuir aos sinais captados um ou mais estados afetivos. Entre as formas de sensoriamento quatro delas se destacam e são representadas na figura 2 :

- A voz (prosódia) (Kopecek, 2000; Tcherkassof, 1999);

- Os observáveis comportamentais, ou seja, as ações do usuário na interface (Bercht \& Viccari, 2000);

- As expressões faciais (Ekman, 1993; Oliveira \& Jaques, 2008; Azcarate, 2005);

- Os sinais fisiológicos - batimentos cardíacos, eletromiograma, tensão muscular, condutividade de pele, respiração (Picard et al, 2001);

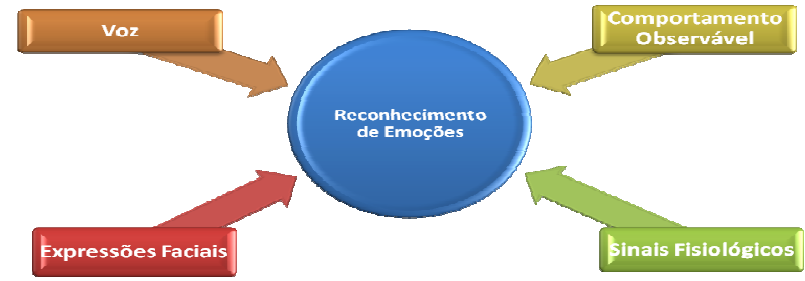

Figura 2 - Sensoriamento de Aspectos Afetivos

Entre as formas de sensoriamento identificadas acima destacaremos os observáveis comportamentais e as expressões faciais, os quais serão o foco deste objeto de estudo.

\section{Os observáveis comportamentais}

Bercht (2001) define por observáveis comportamentais todas as ações de um agente passiveis de serem identificadas por um sistema ou agente artificial e que são consideradas importantes definidoras de comportamentos, conforme a visão do projetista. Em sua proposta de tese ela identifica a motivação em trabalhar com um agente tutor, inferindo fatores de confiança, persistência e independência definidos por (Soldato \& Boulay, 1995) e (Serres, 1993).

Longhi et al (2009) amplia o modelo proposto inicialmente por (Bercht, 2001) e seguindo as categorias afetivas baseadas nas definições de (Scherer, 2005) e utilizando o espaço de Representação de Estados de Ânimo (REA) proposto por (Tran, 2004) e aqui demonstrado na figura 3, para os estados de ânimo: (1) animado, (2) desanimado, (3) satisfeito e (4) insatisfeito consegue inferir estes estados de ânimo em seu protótipo, o Anima-k.

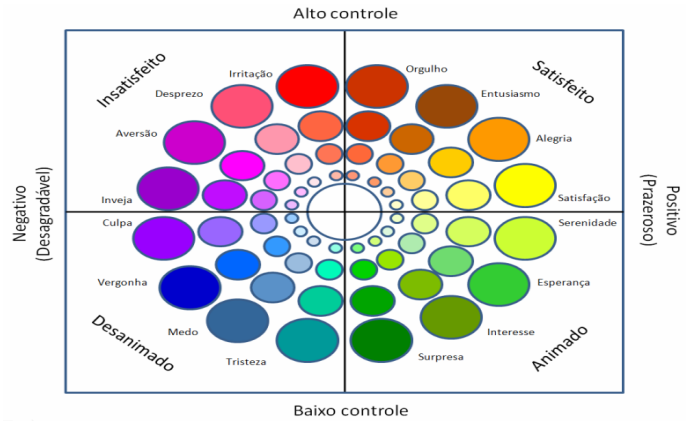

Figura 3 - Espaço de Representação de Estados de Ânimo (Tran, 2004) 
O Anima-k é um objeto de aprendizagem (OA) de lógica de programação feito por (Longhi et al, 2009) baseados nos conceitos de inferência de estados de ânimo. Nessa pesquisa, o uso do OA é precedido de um teste psicológico denominado IFP $^{3}$, onde um psicólogo autorizado recolhe dados sobre os traços de personalidade do aluno, incluindo seis variáveis de interesse: dominância, desempenho, agressão, persistência, mudança e autonomia. Após a realização deste inventário (IFP) o aluno inicia o uso do Anima-k. A REA aparece em três momentos distintos: ao início da tarefa, no meio e em sua conclusão. Nestas três oportunidades o aluno pode indicar ao sistema em qual estado de ânimo ele julga encontrar-se. Ao concluírem suas tarefas, os alunos relatavam textualmente suas sensações sobre a atividade. Visando identificar a manifestação afetiva dos relatos produzidos pelos alunos, técnicas de mineração de textos utilizando a base WordNet Affect $\mathrm{BR}^{4}$ (Pasqualotti, 2008) eram aplicadas. Todos estes dados capturados são submetidos a técnicas de Inteligência Artificial (IA) ${ }^{5}$ para compreender o estado afetivo do aluno.

\section{Uso da webcam na inferência de estados afetivos}

A análise das expressões faciais são um método de sensoriamento que vem despertando um interesse crescente dos pesquisadores. Pesquisas sobre expressões faciais têm como principal alvo a inferência de estados afetivo do usuário (Ekman et al, 2002; Oliveira \& Jaques, 2008; Azcarate, 2005).

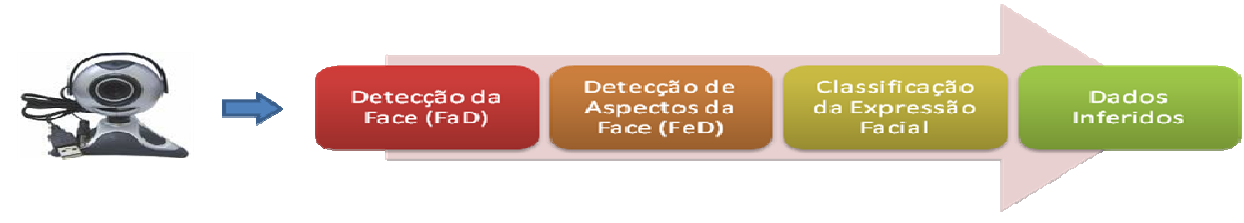

Figura 4 - Passos para reconhecimento de emoções faciais

O naturalista britânico Charles Darwin, escreveu em 1872, um dos livros mais populares sobre a análise das expressões faciais nos seres humanos e sua interação com as emoções (Darwin, 1998). Ele evidencia a expressão de emoções através de expressões faciais como uma das mais significativas formas de comunicação não verbal e aponta que estas formas de expressão podem ser encontradas em diferentes culturas. Esta ultima afirmação é objeto de estudo de (Ekman et al 2002; Ekman, 1993). Como apontado por Darwin, Ekman afirma que parece existir um conjunto de expressões faciais emocionais que são universais, isto é, representam a mesma emoção em diversas culturas. Estas expressões faciais universais são as que representam a alegria, tristeza, fúria, medo, surpresa e nojo.

Para conseguir detectar das emoções são necessários alguns passos intermediários demonstrados na figura 4. Utilizando métodos de Visão Computacional (VC) devemos realizar a detecção de faces (FaD, de Face Detection) e a seguir detecção de características faciais (FeD, de Feature Detection). Mapeadas as características

\footnotetext{
${ }^{3}$ IFP - Inventário Fatorial de Personalidade é uma adaptação de uma versão modificada do Edwards Personal Preference Schedule (EPPS), desenvolvido por Allen L. Edwards em 1953 e 1959, com a finalidade de medir 15 dimensões da personalidade.

${ }^{4}$ WordNet Affect BR mantém um léxico com palavras de domínio afetivo para a língua portuguesa.

${ }^{5}$ No estudo em questão foram utilizadas as redes bayesianas.

V. $7 \mathrm{~N}^{\mathrm{o}}$ 3, dezembro, 2009
} 
necessárias elas são enviadas para classificadores previamente treinados, inferindo a emoção (Oliveira \& Jaques, 2008).

Todas estas etapas estão longe de serem triviais e exigem técnicas de Visão Computacional e Inteligência Artificial. Basicamente existem duas abordagens utilizadas para inferência de emoções por expressões faciais:

Classificação de imagens - este método consiste em treinar um classificador, através de técnicas de machine learning ${ }^{6}$, com milhares de imagens contendo a expressões desejadas e suas respectivas emoções, como por exemplo, haar-like features. Alguns aspectos da imagem devem ser obtidos como os pontos de características faciais (FCPs - Facial Characteristc Points). A figura 5 exibe quais os pontos da face que devem ser identificados.

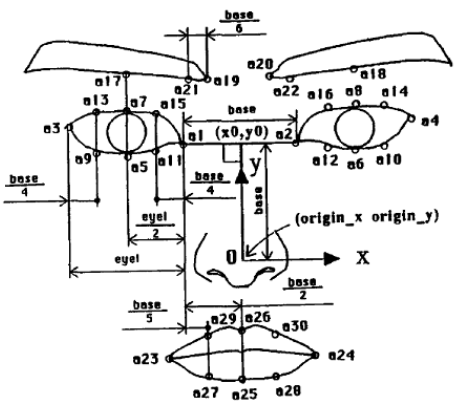

Figura 5 - Pontos de características faciais (Oliveira, 2008)

Uso de um modelo psicológico como o Facial Action Coding System (FACS). FACS é um modelo criado pelos psicólogos Paul Ekman e Wallace V. Friesen que categoriza as aparências faciais causadas por contrações musculares em Unidades de Ação (Action Units - AUs). No modelo foram definidas 44 AUs que podem representar mais de 7000 expressões faciais diferentes. A ocorrência de uma dada emoção pode ser inferida quando a combinação simultânea de certas AUs é detectada na face. Por exemplo, a AU1 representa sobrancelhas levantadas, e a ocorrência das AUs 1, 2, 4, 5 e 25 representam o medo. Estes estudos sobre FACS foram expandidos na década de 80 para a criação de outro modelo, o Emotion FACS - (EMFACS) (Oliveira \& Jaques, 2008).

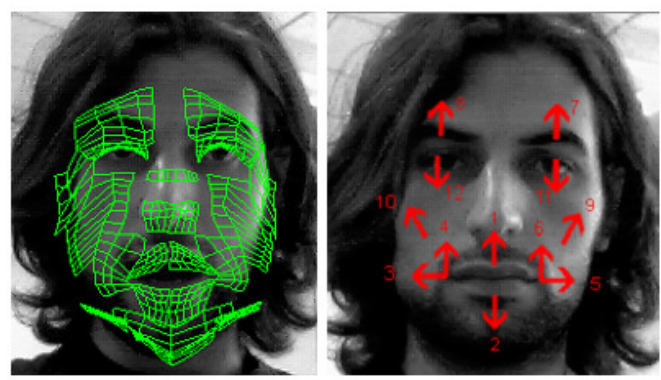

Figura 6 - Ekman "unit actions" (UAs) (Azcarate et al, 2005)

Diversas pesquisas estão sendo realizadas utilizando os métodos acima citados. Oliveira \& Jaques (2008) construíram um classificador que extrai informações de um webcam com intervalos de 3 segundos, analisa as imagens e detecta com

\footnotetext{
${ }^{6}$ Machine Learning - consiste em uma técnica na qual treinamos um sistema com conjunto de termos e sua respectiva resposta. Depois de treinado quando aparecer um problema com termos parecidos a um já treinado o sistema apresentará o resultado.
} 
aproximadamente $60 \%$ de acertos as seis emoções básicas (alegria, medo, raiva, repulsa, surpresa e tristeza), mas tendo $85 \%$ de acerto para alegria, medo e tristeza. (Azcarate et al, 2005) relatam um comparativo entre dois classificadores, o TAN Bayes e o Naive Bayes, ambos baseados na teoria de redes bayesianas, obtendo com o Nayve Bayes uma taxa de $85 \%$ de acerto na detecção da correta emoção.

A Universidade de Amsterdam comercializa um software para detecção de emoções em imagens de webcam (Visual Recognition, 2009) que está sendo testado pelo Grupo de Computação Afetiva da UFRGS, a fim de ser utilizado no apoio ao sensoriamento afetivo de objetos de aprendizagem. O software consegue descobrir através da face do usuário, seis categorias de emoções básicas, e grava em um log para análise. A figura 6 mostra o programa em ação.

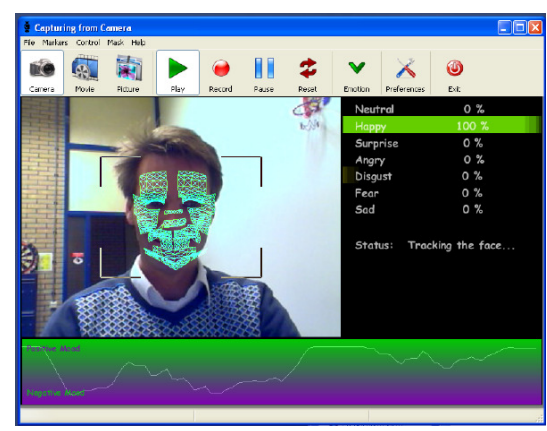

Figura 7 - Visual Recognition

\section{Questões do uso da webcam na educação}

Nas seções anteriores apresentamos alguns estudos onde o uso das webcams na educação parece remeter a uma nova realidade de suporte e melhoria dos ambientes de ensino e aprendizagem. Exemplos do uso desta tecnologia em portadores de necessidade especiais, tarefas de apoio ao ambiente escolar e principalmente no suporte ao sensoriamento dos estados afetivos dos alunos foram apresentados.

Mesmo diante deste novo quadro que desponta, algumas questões éticas devem ser debatidas:

- O que o aluno acha de ter uma câmera de vídeo captando suas reações enquanto este realiza tarefas didáticas;

- O que o professor/tutor pensa de um ambiente repleto de câmeras observando seus métodos e ferramentas de ensino;

- O que pode ser considerado ou não uma invasão de privacidade;

Lembramos que estas questões éticas partem do princípio de que novos OAs podem ser construídos com o uso das webcams como forma de sensoriamento, mas este debate serve para pensarmos em como procedermos para que não haja o uso indevido da tecnologia em questão.

\section{Desafios}

O objetivo destes estudos não se resume em verificar o que hoje existe, mas sim de formular, discutir e pesquisar respostas às questões que se fazem presentes dado as condições tecnológicas hodiernas e suas implicações na educação. Nesta meta pode-se verificar que ainda encontram-se questões não respondidas, como: 
- Os estados afetivos medidos através de ferramentas que utilizem o mapeamento das expressões faciais podem inferir estados cognitivos?

- Como projetar e desenvolver arquitetura eficiente para objetos de aprendizagem (OAs) que utilizem o sensoriamento visual como forma alternativa e/ou complementar de inferência dos estados afetivos?

- O mapeamento on-line dos estados afetivos pode ajudar a melhorar a aprendizagem, o ensino, a motivação e à predisposição em aprender dos alunos quando em uso de OAs?

Sabemos que os caminhos a serem trilhados para a obtenção destas respostas, não são nada triviais, mas as perspectivas dos resultados oriundos da obtenção destas respostas é a grande motivação desta pesquisa.

\section{Referências Bibliográficas}

AZCARATE, Aitor, HAGELOH, Felix, VAN DE SANDE, Koen, VALENTI, Roberto Automatic facial emotion recognition - Universiteit van Amsterdam June 2005

BERCHT, M.; VICCARI, R. Pedagogical agents with affective and cognitive dimensions. In: CONGRESO IBEROAMERICNAO DE INFORMATICA EDUCATIVA, 2000, Vina del Mar. Actas... Santiago: Universidad de Chile, 2000.

BERCHT, M.. Em Direção a Agentes Pedagógicos com Dimensões Afetivas. Instituto de Informática. UFRGS. Tese de Doutorado. Porto Alegre. 2001.

BRUCE, V.; YOUNG, A. - British journal of psychology (London, England: 1953), 1986 ncbi.nlm.nih.gov

BRUSILOVSKY, Peter: Methods and Techniques for Adaptive Hypermedia, 1996.

DAMÁSIO, A., R. O Erro de Descartes. Emoção, Razão e o Cérebro Humano. São Paulo: Companhia das Letras. 1996. 330p.

DARWIN, Charles The Expression of Emotions in Man and Animals (1872) Oxford University Press, USA; 3rd edition (April 9, 1998), 473p.

EKMAN, P. Facial Expression and Emotion. American Psychologist 48, (1993), 384-392.

EKMAN, P., FRIESEN, W.V. and HAGER, J.C. Facial Action Coding System: The manual. Research Nexus division of Network Information Research Corporation, Salt Lake City, Estados Unidos, 2002.

GOLEMAN, D. Emotional Intelligence. New York: Bantam Books, 1995.

LONGHI, Magalí T.; PEREIRA, Daniela Forgiarini; BERCHT, Magda; BEHAR, Patricia Alejandra Um experimento para compreender como os aspectos afetivos podem ser reconhecidos em ambientes virtuais de aprendizagem Revista Novas Tecnologias na Educação Vol.7 Porto Alegre, Junho 2009.

LE DOUX, J. O Cérebro Emocional. Rio de Janeiro: Objetiva, 1998. 332p.

LYRA, Rodrigo, DELGADO, Gabriel A., DAZI, Rudimar L.S. Desenvolvimento de um Jogo Interativo Utilizando WebCam UFPE - Pernambuco 2007

JAQUES, P.A. e VICARI, R.M. Infering Emotions and Applying Affective Tactics for a Better Learning. In: R. M. Vicari, P. A. Jaques, et al (Ed.). Agent-Based Tutoring Systems by Cognitive and Affective Modeling. New York: IGI Global, 2008. Infering Emotions and Applying Affective Tactics for a Better Learning, 2008, p.135-155.

KOPECEK, I. Emotions and Prosody in Dialogues: An Algebraic Approach Based on User Modelling. In: ISCA Workshop on Speech and Emotions. Proceedings... Belfast: ISCA, 2000. p. 184-189.

MSNBC - Microsoft Messenger Journal - Disponível em : $\quad$ http://www.msnbc.msn.com/id/28452074/ Acessado em 21/10/2009.

MARIN, Luciene de Oliveira - Técnicas Estatísticas para Reconhecimento e Detecção de Face Disponível em: http://www.inf.ufsc.br/ visao/2001/luciene/index.html Acesso em 21/10/2009. 
NOZAWA, Erika H., OLIVEIRA, Elaine H.T., VICARI,Rosa M., JÚNIOR, Alberto N.C. Esquema de integração de hipermídias adaptativas em ambientes virtuais de aprendizagem em RENOTE Dez, 2008 .

OLIVEIRA, E. ; JAQUES, Patrícia Augustin. Inferindo as emoções do usuário pela face através de um sistema psicológico de codificação facial. In: Simpósio Brasileiro sobre Fatores Humanos em Sistemas Computacionais, Porto Alegre. : SBC/ACM, 2008. p. 156-165.

PASQUALI, L.; AZEVEDO, M. M. \& GHESTI, I. (1997) Inventário Fatorial de Personalidade: manual técnico e de aplicação. São Paulo: Casa do Psicólogo.

PASQUALOTTI, P. R. Reconhecimento de expressões de emoções na interação mediada por computador. Computação Aplicada. UNISINOS, Dissertação de Mestrado. São Leopoldo, 2008.

PENTEADO, Bruno Elias ; MARANA, A. N. . A Video-Based Biometric Authentication for ELearning Web Applications. In: Eleventh International Conference on Enterprise Information Systems, ICEIS 2008, 2009, Milão. LNBIP - Lecture Notes on Business Information Processing. Berlin : SpringerVerlag, 2009. v. 24. p. 770-779.

PENTEADO, Bruno Elias ; MARANA, A. N. . Aluno de mestrado é premiado na Itália. http://www.fc.unesp.br/noticiencias/materia/838, 02 jun. 2009.

PIAGET, J. Les relations entre l'intelligence et l'affectivité dans le developpement de l'enfant. In : RIMÉ, B.; SCHERER, K. (Ed.). Les Émotions. Textes de base en psychologie . Paris: Delachaux et Niestlé, 1989. p. 75-95.

PICARD, R. W. Affective Computing. Cambridge: MIT Press. 1997.

PICARD, P.; VYZAS, E.; HEALEY, J. Toward Machine Emotional Intelligence: Analysis of Affective Physiological State. IEEE Transactions Pattern Analysis and Machine Intelligence, Los Alamitos, v. 23 , n. 10, 2001.

SERRES, M. Filosofia mestiça. Rio de Janeiro : Nova Fronteira, 1993.

SCHERER, K. What are emotions? And how can they be measured? In: Social Science Information 44 (4), 695-729. 2005.

SCHERER, K. R., \& TRAN, V. Effects of emotion on the process of Organization Learning. In I. Nonaka (Ed.), Handbook of organizational learning and knowledge (pp. 369-392). Oxford, UK: Oxford University Press. 2001.

SOLDATO, T., \& BOULAY,B. Implementation of Motivational Tactics in Tutoring Systems. Journal of Artificial Intelligence in Education, Charlottesville,v.6, p.337-378, 1995.

TRAN, V. The influence of emotions on decision-making processes in management teams. (L'influence des Emotions sur les Processus de Prise de Décision dans les Equipes de Cadres). Faculte De Psychologie et des Sciences de l'Education. Université de Genève. Thèse No 323. 2004.

TCHERKASSOF, A. Les indices de préparation à l'action et la reconnaissance dês expressions émotionnelles faciales. Revue Européenne de Psychologie Appliquée, [S. 1.], v.49, n. 2, p. 99-105, 1999.

UEPG - Universidade Federal de Ponta Grossa - Apresenta o projeto leitor em braile via webcam Disponível em: http://www.jornalcomunicacao.ufpr.br/node/6965 . Acesso em: 21 out. 2009.

VAIL, P. Emotion: The On/Off Switch for Learning. Rosemont, NJ: Modern Learning Press, 1994.

VIEIRA, Tiago F. , FONTANA, Eduardo Dispositivo de rastreamento de movimentos oculares baseado em webcam e iluminação com led infravermelho - Anais $21^{\circ}$ Congresso Brasileiro de Engenharia Biomédica p.669-672. Salvador (BA) 2008

VISUAL RECOGNITION Software para reconhecimento de emoções através de expressões faciais. Disponível em http://www.visual-recognition.nl/ Acesssado em 21/10/2009

VYGOTSKY, Lev S. A formação social da mente. São Paulo: Martins Fontes, 1999.191p.

WHITEHILL, Jacob; BARTLETT, Marian; MOVELLAN, Javier - Measuring the Perceived Dificulty of a Lecture Using Automatic Facial Expression Recognition - MPLab TR , University of California, San Diego 2008 - Disponível em http://www.physorg.com/news133590374.html . Acesso em 21/10/2009. 